\title{
EXISTENCE AND STABILITY OF PERIODIC SOLUTIONS OF A RAYLEIGH TYPE EQUATION
}

\author{
YONG WANG ${ }^{\otimes}$ and XIAN-ZHI DAI
}

(Received 14 May 2008)

\begin{abstract}
In this work, we shall be concerned with the following forced Rayleigh type equation:

$$
\left.x^{\prime \prime}(t)+f\left(x^{\prime}(t)\right)+g(t, x(t))\right)=e(t) .
$$

Under certain assumptions, some criteria for guaranteeing the existence, uniqueness and asymptotic stability (in the Lyapunov sense) of periodic solutions of this equation are presented by applying the Manásevich-Mawhin continuation theorem, Floquet theory, Lyapunov stability theory and some analysis techniques. Moreover, an example is provided to demonstrate the applications of our results.
\end{abstract}

2000 Mathematics subject classification: primary 34K15, 34C25.

Keywords and phrases: Rayleigh equation, periodic solution, existence, uniqueness, stability.

\section{Introduction}

In this note we consider the existence, uniqueness and asymptotic stability of periodic solutions of the following forced Rayleigh type equation:

$$
x^{\prime \prime}(t)+f\left(x^{\prime}(t)\right)+g(t, x(t))=e(t),
$$

where $f \in C^{2}(\mathbb{R}, \mathbb{R}), e \in C(\mathbb{R}, \mathbb{R}), g \in C^{1}\left(\mathbb{R}^{2}, \mathbb{R}\right), e(t)$ and $g(t, x)$ are $T$-periodic functions in $t$ with $T>0$, and $f(0)=0$.

It is well known that the British mathematical physicist Lord Rayleigh (John William Strutt, 1842-1919), a Nobel Prize Laureate in Physics in 1904, introduced an equation of the form

$$
x^{\prime \prime}(t)+f\left(x^{\prime}(t)\right)+a x(t)=0
$$

to model the oscillations of a clarinet reed; for details, see [16]. One of the most difficult problems connected with the study of the Rayleigh equation (1.2) is whether

This work is supported by SWPU Science and Technology Fund, China.

(C) 2009 Australian Mathematical Society 0004-9727/2009 \$16.00 
it can support limit cycles. Limit cycles are very important in science. They model systems that exhibit self-sustained oscillations. In other words, these systems oscillate even in the absence of external periodic forcing.

During the past several years, many authors have studied the self-sustained oscillations in acoustics. Fletcher [3] showed that there exist particular ranges of acoustic impedance for the inlet and outlet ducts to the valve within which selfsustained valve oscillation is possible. Keith and Rand [6], Shimizu and Morioka [15] and Shimizu [14] investigated the existence conditions for limit cycles of the Rayleigh equation, that is, conditions under which auto-oscillations occur. The question of periodic solutions was also studied for different generalizations of the Rayleigh equation, for example (1.1). In [4], Gaines and Mawhin introduced some continuation theorems and applied them in discussing the existence of solutions of certain ordinary differential equations. In particular, a specific example was provided in [4, p. 99] on how $T$-periodic solutions could be obtained by means of these theorems for (1.1) with $e(t)=0$. In the course of derivations, it was realized that once a priori bounds for the $T$-periodic solutions of the homotopic equations is known, then standard procedures will allow these theorems to imply the existence of $T$-periodic solutions to the original equation. Applying these approaches, the authors of $[8-10,17,18]$ continued to discuss the Rayleigh equation and obtained many new results on the existence of $T$-periodic solutions of (1.1), and generalized some results in [4]. Other authors researched the stability of periodic solutions of the Duffing type equations

$$
x^{\prime \prime}(t)+b x^{\prime}(t)+g(t, x(t))=0
$$

and

$$
x^{\prime \prime}(t)+b x^{\prime}(t)+g(t, x(t))=e(t)
$$

In [13], Ortega studied the stability of periodic solutions of (1.3) and obtained some stability results by topological index. Then Lazer and McKenna [7] obtained stability results by converting (1.3) to a fixed-point problem. Recently, Chen and Li [1] studied the rate of decay of stable periodic solutions of (1.4) and determined a sharp rate of exponential decay for a solution that is near to the unique periodic solution. However, to our knowledge, there are much fewer results on the stability of periodic solutions of (1.1). Hence it is essential to continue to study periodic solutions of (1.1) in this case.

The main purpose of this paper is to establish some sufficient conditions for guaranteeing the existence, uniqueness and asymptotic stability of $T$-periodic solutions of (1.1), by applying the Manásevich-Mawhin continuation theorem, Floquet theory, Lyapunov stability theory and some analysis techniques.

The following notation will be used throughout the rest of this paper.

$$
|x|_{\infty}=\max _{t \in[0, T]}|x(t)|, \quad\left|x^{\prime}\right|_{\infty}=\max _{t \in[0, T]}\left|x^{\prime}(t)\right|, \quad|x|_{k}=\left(\int_{0}^{T}|x(t)|^{k} d t\right)^{1 / k} .
$$

Set

$$
C_{T}^{1}:=\left\{x \in C^{1}(\mathbb{R}, \mathbb{R}) \mid x(t+T)=x(t)\right\}
$$


and

$$
C_{T}:=\{x \in C(\mathbb{R}, \mathbb{R}): x(t+T)=x(t)\},
$$

which are two Banach spaces with the norms

$$
\|x\|_{C_{T}^{1}}=\max \left\{|x|_{\infty},\left|x^{\prime}\right|_{\infty}\right\}, \quad\|x\|_{C_{T}}=|x|_{\infty} .
$$

Our main theorem is as follows.

THEOREM 1.1. Assume that there exist constants $C_{1} \geq 0, C_{2} \geq 0$ and $D_{1} \geq 0$ such that:

$\left(\mathrm{H}_{1}\right)(g(t, u)-g(t, v))(u-v)>0$, for all $t, u, v \in \mathbb{R}$ with $u \neq v$;

$\left(\mathrm{H}_{2}\right)|f(t, u)-f(t, v)| \leq C_{1}|u-v|$, for all $t, u, v \in \mathbb{R}$;

$\left(\mathrm{H}_{3}\right)|g(t, u)-g(t, v)| \leq C_{2}|u-v|$, for all $t, u, v \in \mathbb{R}$;

$\left(\mathrm{H}_{4}\right)\left(C_{1} / 2 \pi\right) T+\left(C_{2} / 4 \pi\right) T^{2}<1$;

$\left(\mathrm{H}_{5}\right) x(g(t, x)-e(t))>0$, for all $|x| \geq D_{1}$ and $t \in \mathbb{R}$;

$\left(\mathrm{H}_{6}\right) \int_{0}^{T}\left[g_{x}^{\prime}(t, x)-\frac{1}{4}\left(f^{\prime}(y)\right)^{2}\right] d t>0$ and $f^{\prime}(y)>0$, for $x \in I_{1}$ and $y \in I_{2}$;

$\left(\mathrm{H}_{7}\right)$

$$
\left\{g_{x}^{\prime}(t, x)-\frac{1}{2} f^{\prime \prime}(y)[e(t)-f(y)-g(t, x)]-\frac{1}{4}\left(f^{\prime}(y)\right)^{2}\right\}<\frac{1}{T^{2}},
$$

for $t \in \mathbb{R}, x \in I_{1}$ and $y \in I_{2} ;$ where

$$
\begin{gathered}
G=\max _{t \in[0, T]}|g(t, 0)|, \quad M_{0}=\frac{\left(G+|e|_{\infty}+C_{2} D_{1}\right) \sqrt{T}}{1-\left(C_{1} / 2 \pi\right) T-\left(C_{2} / 4 \pi\right) T^{2}}, \\
M_{1}=D_{1}+\frac{\sqrt{T^{3}}}{4 \pi} M_{0}, \quad M_{2}=\frac{1}{2} \sqrt{T} M_{0}, \\
I_{1}=\left[-M_{1}, M_{1}\right] \quad \text { and } \quad I_{2}=\left[-M_{2}, M_{2}\right] .
\end{gathered}
$$

Then (1.1) has a unique T-periodic solution which is asymptotically stable.

In Section 2 we introduce some lemmas which will help us to obtain the main theorem, the proof of which is given in Section 3. The paper concludes with an illustrative example is provided to demonstrate the application of our results.

\section{Lemmas}

In this section, we shall introduce some lemmas which will help us to obtain our main theorem. First, let us recall the Manásevich-Mawhin continuation theorem, which is useful in obtaining the existence of $T$-periodic solutions of (1.1).

For the periodic boundary value problem

$$
\left(\varphi_{p}\left(x^{\prime}(t)\right)\right)^{\prime}=h\left(t, x, x^{\prime}\right), \quad x(0)=x(T), \quad x^{\prime}(0)=x^{\prime}(T),
$$

where $p>1, \varphi_{p}: \mathbb{R} \rightarrow \mathbb{R}, \varphi_{p}(s)=|s|^{p-2} s$ is a one-dimensional $p$-Laplacian, and $h \in C\left(\mathbb{R}^{3}, \mathbb{R}\right)$ is $T$-periodic in the first variable, we have the following result. 
LEMMA 2.1. [11] Let $\Omega$ be an open bounded set in $C_{T}^{1}$. Suppose the following conditions hold:

(i) for each $\lambda \in(0,1)$ the problem

$$
\left(\varphi_{p}\left(x^{\prime}(t)\right)\right)^{\prime}=\lambda h\left(t, x, x^{\prime}\right), \quad x(0)=x(T), \quad x^{\prime}(0)=x^{\prime}(T)
$$

has no solution on $\partial \Omega$;

(ii) the equation

$$
F(a):=\frac{1}{T} \int_{0}^{T} h(t, a, 0) d t=0
$$

has no solution on $\partial \Omega \cap \mathbb{R}$;

(iii) the Brouwer degree of $F$ is

$$
\operatorname{deg}(F, \Omega \cap \mathbb{R}, 0) \neq 0 .
$$

Then the periodic boundary value problem (2.1) has at least one T-periodic solution on $\bar{\Omega}$.

REMARK 2.2. In particular, if $p=2$, (2.1) reduces to the following periodic boundary value problem:

$$
x^{\prime \prime}(t)=h\left(t, x, x^{\prime}\right), \quad x(0)=x(T), \quad x^{\prime}(0)=x^{\prime}(T),
$$

which implies that the above Lemma 2.1 can be used to investigate the existence of $T$-periodic solutions of (1.1).

Next, two lemmas will be introduced to help us in obtaining the uniqueness of $T$-periodic solutions of (1.1).

LEMMA 2.3. If $x \in C^{2}(\mathbb{R}, \mathbb{R})$ with $x(t+T)=x(t)$, then

$$
\left|x^{\prime}\right|_{2}^{2} \leq\left(\frac{T}{2 \pi}\right)^{2}\left|x^{\prime \prime}\right|_{2}^{2} .
$$

PROOF. Lemma 2.3 is a direct consequence of the Wirtinger inequality; see $[5,12]$ for its proof.

Lemma 2.4. Suppose $\left(H_{1}\right)-\left(H_{4}\right)$ hold. Then (1.1) has at most one T-periodic solution.

PROOF. Suppose that $x_{1}(t)$ and $x_{2}(t)$ are two $T$-periodic solutions of (1.1). Then

$$
\left[x_{1}(t)-x_{2}(t)\right]^{\prime \prime}+\left[f\left(x_{1}^{\prime}(t)\right)-f\left(x_{2}^{\prime}(t)\right)\right]+\left[g \left(t, x_{1}(t)-g\left(t, x_{2}(t)\right]=0 .\right.\right.
$$

Setting $Z(t)=x_{1}(t)-x_{2}(t)$, we obtain, from (2.3), that

$$
Z^{\prime \prime}(t)+\left[f\left(x_{1}^{\prime}(t)\right)-f\left(x_{2}^{\prime}(t)\right)\right]+\left[g \left(t, x_{1}(t)-g\left(t, x_{2}(t)\right]=0 .\right.\right.
$$


Since $Z(t)=x_{1}(t)-x_{2}(t)$ is a continuous $T$-periodic function in $\mathbb{R}$, there exist two constants $t_{\max }, t_{\min } \in \mathbb{R}$ such that

$$
Z\left(t_{\max }\right)=\max _{t \in[0, T]} Z(t)=\max _{t \in \mathbb{R}} Z(t), \quad Z\left(t_{\min }\right)=\min _{t \in[0, T]} Z(t)=\min _{t \in \mathbb{R}} Z(t) .
$$

It follows that

$$
Z^{\prime}\left(t_{\max }\right)=x_{1}^{\prime}\left(t_{\max }\right)-x_{2}^{\prime}\left(t_{\max }\right)=0, \quad Z^{\prime \prime}\left(t_{\max }\right) \leq 0
$$

and

$$
Z^{\prime}\left(t_{\min }\right)=x_{1}^{\prime}\left(t_{\min }\right)-x_{2}^{\prime}\left(t_{\min }\right)=0, \quad Z^{\prime \prime}\left(t_{\min }\right) \geq 0 .
$$

Then, by (2.4)-(2.7),

$$
\begin{aligned}
g\left(t_{\max }, x_{1}\left(t_{\max }\right)\right)-g\left(t_{\max }, x_{2}\left(t_{\max }\right)\right) & =-Z^{\prime \prime}\left(t_{\max }\right)-\left[f\left(x_{1}^{\prime}\left(t_{\max }\right)\right)-f\left(x_{2}^{\prime}\left(t_{\max }\right)\right)\right] \\
& =-Z^{\prime \prime}\left(t_{\max }\right) \geq 0
\end{aligned}
$$

and

$$
\begin{aligned}
g\left(t_{\min }, x_{1}\left(t_{\min }\right)\right)-g\left(t_{\min }, x_{2}\left(t_{\min }\right)\right) & =-Z^{\prime \prime}\left(t_{\min }\right)-\left[f\left(x_{1}^{\prime}\left(t_{\min }\right)\right)-f\left(x_{2}^{\prime}\left(t_{\min }\right)\right)\right] \\
& =-Z^{\prime \prime}\left(t_{\min }\right) \leq 0
\end{aligned}
$$

From (2.8) and (2.9), we know that there exists a constant $t_{0} \in \mathbb{R}$ such that

$$
g\left(t_{0}, x_{1}\left(t_{0}\right)\right)-g\left(t_{0}, x_{2}\left(t_{0}\right)\right)=0 .
$$

Now set $t_{0}=n T+\tilde{t}_{0}$, where $\tilde{t}_{0} \in[0, T]$ and $n$ is an integer. Note that since $Z(t+T)=Z(t)$, we get, by $\left(\mathrm{H}_{1}\right)$ and (2.10), that

$$
Z\left(\tilde{t}_{0}\right)=Z\left(t_{0}\right)=x_{1}\left(t_{0}\right)-x_{2}\left(t_{0}\right)=0 .
$$

Hence, for any $t \in\left[\tilde{t}_{0}, \tilde{t}_{0}+T\right]$, we obtain

$$
|Z(t)|=\left|Z\left(\tilde{t}_{0}\right)+\int_{\tilde{t}_{0}}^{t} Z^{\prime}(s) d s\right| \leq \int_{\tilde{t}_{0}}^{t}\left|Z^{\prime}(s)\right| d s
$$

and

$$
|Z(t)|=\left|Z\left(\tilde{t}_{0}+T\right)+\int_{\tilde{t}_{0}+T}^{t} Z^{\prime}(s) d s\right|=\left|-\int_{t}^{\tilde{t}_{0}+T} Z^{\prime}(s) d s\right| \leq \int_{t}^{\tilde{t}_{0}+T}\left|Z^{\prime}(s)\right| d s .
$$

Now combining above two inequalities, we get

$$
|Z(t)| \leq \frac{1}{2} \int_{0}^{T}\left|Z^{\prime}(s)\right| d s
$$


This, together with the Schwarz inequality, gives

$$
|Z|_{\infty}=\max _{t \in\left[\tilde{t}_{0}, \tilde{t}_{0}+T\right]}|Z(t)| \leq \frac{1}{2} \int_{0}^{T}\left|Z^{\prime}(s)\right| d s \leq \frac{1}{2}|1|_{2}\left|Z^{\prime}\right|_{2}=\frac{1}{2} \sqrt{T}\left|Z^{\prime}\right|_{2} .
$$

Next, multiplying $Z^{\prime \prime}(t)$ and (2.4) and integrating it from 0 to $T$, we obtain, by Lemma 2.3, $\left(\mathrm{H}_{2}\right),\left(\mathrm{H}_{3}\right),(2.12)$ and the Schwarz inequality, that

$$
\begin{aligned}
\left|Z^{\prime \prime}\right|_{2}^{2}= & -\int_{0}^{T}\left[f\left(x_{1}^{\prime}(t)\right)-f\left(x_{2}^{\prime}(t)\right)\right] Z^{\prime \prime}(t) d t \\
& -\int_{0}^{T}\left[g\left(t, x_{1}(t)\right)-g\left(t, x_{2}(t)\right)\right] Z^{\prime \prime}(t) d t \\
\leq & \int_{0}^{T}\left|f\left(x_{1}^{\prime}(t)\right)-f\left(x_{2}^{\prime}(t)\right)\right|\left|Z^{\prime \prime}(t)\right| d t \\
& +\int_{0}^{T}\left|g\left(t, x_{1}(t)\right)-g\left(t, x_{2}(t)\right)\right|\left|Z^{\prime \prime}(t)\right| d t \\
\leq & \int_{0}^{T} C_{1}\left|x_{1}^{\prime}(t)-x_{2}^{\prime}(t)\right|\left|Z^{\prime \prime}(t)\right| d t+\int_{0}^{T} C_{2}\left|x_{1}(t)-x_{2}(t)\right|\left|Z^{\prime \prime}(t)\right| d t \\
\leq & \int_{0}^{T} C_{1}\left|Z^{\prime}(t)\right|\left|Z^{\prime \prime}(t)\right| d t+\int_{0}^{T} C_{2}|Z(t)|\left|Z^{\prime \prime}(t)\right| d t \\
\leq & C_{1}\left|Z^{\prime}\right|_{2}\left|Z^{\prime \prime}\right|_{2}+C_{2} \sqrt{T}|Z|_{\infty}\left|Z^{\prime \prime}\right|_{2} \\
\leq & \left(\frac{C_{1}}{2 \pi} T+\frac{C_{2}}{4 \pi} T^{2}\right)\left|Z^{\prime \prime}\right|_{2}^{2} .
\end{aligned}
$$

Since $Z(t), Z^{\prime}(t)$ and $Z^{\prime \prime}(t)$ are continuous $T$-periodic functions, we get, by Lemma 2.3, $\left(\mathrm{H}_{4}\right)$ and $(2.12)$, that

$$
Z(t)=Z^{\prime}(t)=Z^{\prime \prime}(t)=0 \quad \text { for all } t \in \mathbb{R},
$$

which implies that $x_{1}(t) \equiv x_{2}(t)$ for all $t \in \mathbb{R}$. Hence, (1.1) has at most one $T$-periodic solution. This completes the proof.

Moreover, consider the homotopic equation of (1.1),

$$
x^{\prime \prime}(t)+\lambda f\left(x^{\prime}(t)\right)+\lambda g(t, x(t))=\lambda e(t), \quad \lambda \in(0,1) .
$$

The following lemma will show that the set of all possible $T$-periodic solutions of (2.14) are bounded in $C_{T}^{1}$ under some restrictive conditions. This result will help us to obtain the existence of $T$-periodic solutions of (1.1) when applying the above Manásevich-Mawhin continuation theorem.

Lemma 2.5. Suppose that $\left(\mathrm{H}_{2}\right)-\left(\mathrm{H}_{5}\right)$ hold. Then the set of $\mathrm{T}$-periodic solutions of (2.14) are bounded in $C_{T}^{1}$. 
PROOF. Let $S \subset C_{T}^{1}$ be the set of $T$-periodic solutions of (2.14). If $S=\emptyset$, the proof is done. Suppose that $S \neq \emptyset$, and let $x \in S$. Then there exist two constants $\bar{t}, \underline{t} \in \mathbb{R}$ such that

$$
x(\bar{t})=\max _{t \in \mathbb{R}} x(t) \quad \text { and } \quad x(\underline{t})=\min _{t \in \mathbb{R}} x(t),
$$

implying that

$$
x^{\prime}(\bar{t})=0, x^{\prime \prime}(\bar{t}) \leq 0 ; \quad x^{\prime}(\underline{t})=0, x^{\prime \prime}(\underline{t}) \geq 0 .
$$

Note that since $f(0)=0$, we obtain, by (2.14) and (2.15), that

$$
g(\bar{t}, x(\bar{t}))-e(\bar{t}) \geq 0 \quad \text { and } \quad g(\underline{t}, x(\underline{t}))-e(\underline{t}) \leq 0
$$

which implies that there exists a constant $\hat{t} \in \mathbb{R}$ such that

$$
g(\hat{t}, x(\hat{t}))-e(\hat{t})=0 .
$$

Subsequently, set $\hat{t}=m T+\hat{t}_{0}$, where $\hat{t}_{0} \in[0, T]$ and $m$ is an integer. Then we get, by $\left(\mathrm{H}_{5}\right)$ and (2.16), that

$$
\left|x\left(\hat{t}_{0}\right)\right|=|x(\hat{t})| \leq D_{1} .
$$

Hence, for any $t \in\left[\hat{t}_{0}, \hat{t}_{0}+T\right]$,

$$
|x(t)|=\left|x\left(\hat{t}_{0}\right)+\int_{\hat{t}_{0}}^{t} x^{\prime}(s) d s\right| \leq D_{1}+\int_{\hat{t}_{0}}^{t}\left|x^{\prime}(s)\right| d s
$$

and

$$
\begin{aligned}
|x(t)| & =\left|x\left(\hat{t}_{0}+T\right)+\int_{\hat{t}_{0}+T}^{t} x^{\prime}(s) d s\right| \leq D_{1}+\left|-\int_{t}^{\hat{t}_{0}+T} x^{\prime}(s) d s\right| \\
& \leq D_{1}+\int_{t}^{\hat{t}_{0}+T}\left|x^{\prime}(s)\right| d s .
\end{aligned}
$$

Now combining above two inequalities, we get

$$
|x(t)| \leq D_{1}+\frac{1}{2} \int_{0}^{T}\left|x^{\prime}(s)\right| d s .
$$

This together with the Schwarz inequality gives

$$
\begin{aligned}
|x|_{\infty} & =\max _{t \in\left[\hat{t}_{0}, \hat{t}_{0}+T\right]}|x(t)| \leq D_{1}+\frac{1}{2} \int_{0}^{T}\left|x^{\prime}(s)\right| d s \\
& \leq D_{1}+\frac{1}{2}|1|_{2}\left|x^{\prime}\right|_{2}=D_{1}+\frac{1}{2} \sqrt{T}\left|x^{\prime}\right|_{2} .
\end{aligned}
$$


Note that, since $f(0)=0$, we obtain, by Lemma 2.3, $\left(\mathrm{H}_{2}\right),\left(\mathrm{H}_{3}\right),(2.14),(2.18)$ and the Schwarz inequality, that

$$
\begin{aligned}
\left|x^{\prime \prime}\right|_{2}^{2}= & -\lambda \int_{0}^{T} f\left(x^{\prime}(t)\right) x^{\prime \prime}(t) d t-\lambda \int_{0}^{T} g(t, x(t)) x^{\prime \prime}(t) d t+\lambda \int_{0}^{T} e(t) x^{\prime \prime}(t) d t \\
= & -\lambda \int_{0}^{T}\left[f\left(x^{\prime}(t)\right)-f(0)\right] x^{\prime \prime}(t) d t \\
& -\lambda \int_{0}^{T}[g(t, x(t))-g(t, 0)+g(t, 0)] x^{\prime \prime}(t) d t+\lambda \int_{0}^{T} e(t) x^{\prime \prime}(t) d t \\
\leq & \int_{0}^{T}\left|f\left(x^{\prime}(t)\right)-f(0)\right|\left|x^{\prime \prime}(t)\right| d t+\int_{0}^{T}|g(t, x(t))-g(t, 0)|\left|x^{\prime \prime}(t)\right| d t \\
& +\int_{0}^{T}|g(t, 0)|\left|x^{\prime \prime}(t)\right| d t+\int_{0}^{T}|e(t)|\left|x^{\prime \prime}(t)\right| \mid d t \\
\leq & \int_{0}^{T} C_{1}\left|x^{\prime}(t)\right|\left|x^{\prime \prime}(t)\right| d t+\int_{0}^{T} C_{2}|x(t)|\left|x^{\prime \prime}(t)\right| d t \\
& +G \int_{0}^{T}\left|x^{\prime \prime}(t)\right| d t+|e|_{\infty} \int_{0}^{T}\left|x^{\prime \prime}(t)\right| d t \\
\leq & C_{1}\left|x^{\prime}\right|_{2}\left|x^{\prime \prime}\right|_{2}^{2}+C_{2} \sqrt{T}|x|_{\infty}\left|x^{\prime \prime}\right|_{2}+\left(G+|e|_{\infty}\right)|1|_{2}\left|x^{\prime \prime}\right|_{2} \\
\leq & \frac{C_{1}}{2 \pi} T\left|x^{\prime \prime}\right|_{2}^{2}+C_{2} \sqrt{T}\left(D_{1}+\frac{1}{2} \sqrt{T}\left|x^{\prime}\right|_{2}\right)\left|x^{\prime \prime}\right|_{2}+\left(G+|e|_{\infty}\right) \sqrt{T}\left|x^{\prime \prime}\right|_{2} \\
\leq & \left(\frac{C_{1}}{2 \pi} T++\frac{C_{2}}{4 \pi} T^{2}\right)\left|x^{\prime \prime}\right|_{2}^{2}+\left(G+|e|_{\infty}+C_{2} D_{1}\right) \sqrt{T}\left|x^{\prime \prime}\right|_{2},
\end{aligned}
$$

where $G=\max _{t \in[0, T]}|g(t, 0)|$. Now, by $\left(\mathrm{H}_{4}\right)$ and (2.19),

$$
\left|x^{\prime \prime}\right|_{2} \leq \frac{\left(G+|e|_{\infty}+C_{2} D_{1}\right) \sqrt{T}}{1-\left(C_{1} / 2 \pi\right) T-\left(C_{2} / 4 \pi\right) T^{2}}=M_{0} .
$$

Since $x(0)=x(T)$, there exists a constant $\tilde{t} \in[0, T]$ such that $x^{\prime}(\tilde{t})=0$. Then for any $t \in[\tilde{t}, \tilde{t}+T]$,

$$
\left|x^{\prime}(t)\right|=\left|x^{\prime}(\tilde{t})+\int_{\tilde{t}}^{t} x^{\prime \prime}(s) d s\right| \leq \int_{\tilde{t}}^{t}\left|x^{\prime \prime}(s)\right| d s
$$

and

$$
\left|x^{\prime}(t)\right|=\left|x^{\prime}(\tilde{t}+T)+\int_{\tilde{t}+T}^{t} x^{\prime \prime}(s) d s\right| \leq\left|-\int_{t}^{\tilde{t}+T} x^{\prime \prime}(s) d s\right| \leq \int_{t}^{\tilde{t}+T}\left|x^{\prime \prime}(s)\right| d s .
$$

Now combining above two inequalities, we get, by the Schwarz inequality, that

$$
\left|x^{\prime}(t)\right| \leq \frac{1}{2} \int_{0}^{T}\left|x^{\prime \prime}(s)\right| d s \leq \frac{1}{2}|1|_{2}\left|x^{\prime \prime}\right|_{2}=\frac{1}{2} \sqrt{T}\left|x^{\prime \prime}\right|_{2},
$$


which, together with (2.20), implies that

$$
\left|x^{\prime}\right|_{\infty}=\max _{t \in[\tilde{t}, \tilde{t}+T]}\left|x^{\prime}(t)\right| \leq \frac{1}{2} \sqrt{T}\left|x^{\prime \prime}\right|_{2}=\frac{1}{2} \sqrt{T} M_{0}=M_{2} .
$$

Moreover, according to Lemma 2.3, (2.18) and (2.20),

$$
|x|_{\infty} \leq D_{1}+\frac{1}{2} \sqrt{T}\left|x^{\prime}\right|_{2} \leq D_{1}+\frac{\sqrt{T^{3}}}{4 \pi}\left|x^{\prime \prime}\right|_{2} \leq D_{1}+\frac{\sqrt{T^{3}}}{4 \pi} M_{0}=M_{1} .
$$

Let $M=\max \left\{M_{1}, M_{2}\right\}$; then we know that

$$
|x|_{\infty} \leq M \quad \text { and } \quad\left|x^{\prime}\right|_{\infty} \leq M
$$

This completes the proof.

REMARK 2.6. According to the proof of Lemma 2.5, we can easily conclude that, if $x(t)$ is a $T$-periodic solution of (1.1), then (2.21) and (2.22) also hold under the conditions of Lemma 2.5 .

Finally, for convenience of use, we recall a well-known principle of linearized stability for periodic systems; for details, see for example [2, pp. 321-322]. Consider the periodic boundary value problem

$$
\left\{\begin{array}{l}
x^{\prime}=F(t, x), \\
x(0)=x(T),
\end{array}\right.
$$

where $F:[0, T] \times \mathbb{R}^{n} \rightarrow \mathbb{R}^{n}$ is a continuous function that is $T$-periodic in $t$ and has continuous first-order partial derivative with respect to $x$. Let $p_{0}$ be a $T$-periodic solution of (2.23); then we associate the $T$-periodic solution $p_{0}$ with the linearized equation

$$
y^{\prime}=F_{x}^{\prime}\left(t, p_{0}\right) y .
$$

The following theorem, which is due to Lyapunov (see [3, Theorem 2.1, p. 322]), shows the connections between the asymptotic stability of the $T$-periodic solution $p_{0}$ of (2.23) and the characteristic exponents of (2.24).

LEMMA 2.7. If the characteristic exponents associated with (2.24) all have negative real parts, then the $T$-periodic solution $p_{0}$ of (2.23) is asymptotically stable.

\section{Proof of Theorem 1.1}

Now we are in the position to present the proof of Theorem 1.1. The proof falls naturally in two parts. We begin by considering a existence and uniqueness result, before turning to asymptotic stability.

According to Remark 2.2, Lemma 2.1 can be used to obtaini the existence of $T$-periodic solutions of (1.1). Set

$$
h\left(t, x, x^{\prime}\right)=e(t)-f\left(x^{\prime}\right)-g(t, x) .
$$


Then (2.14) is equivalent to

$$
x^{\prime \prime}(t)=\lambda h\left(t, x(t), x^{\prime}(t)\right), \quad \lambda \in(0,1) .
$$

By Lemma 2.5, there exists $M_{3}>M$ such that, for any $T$-periodic solution $x(t)$ of (2.14) or (3.2),

$$
|x|_{\infty}<M_{3} \text { and } \quad\left|x^{\prime}\right|_{\infty}<M_{3}
$$

Let

$$
\Omega=\left\{x \in C_{T}^{1}:|x|_{\infty}<M_{3},\left|x^{\prime}\right|_{\infty}<M_{3}\right\} .
$$

Then we know that (3.2) has no solution on $\partial \Omega$ as $\lambda \in(0,1)$, so condition (i) of Lemma 2.1 is satisfied.

On the other hand, since

$$
F(a):=\frac{1}{T} \int_{0}^{T} h(t, a, 0) d t
$$

for any $x \in \partial \Omega \cap \mathbb{R}, x=M_{3}$ or $x=-M_{3}$, and $f(0)=0$, we obtain, by $\left(\mathrm{H}_{5}\right)$, that

$$
\begin{gathered}
F\left(M_{3}\right)=\frac{1}{T} \int_{0}^{T}\left[e(t)-g\left(t, M_{3}\right)\right] d t<0, \\
F\left(-M_{3}\right)=\frac{1}{T} \int_{0}^{T}\left[e(t)-g\left(t,-M_{3}\right)\right] d t>0,
\end{gathered}
$$

which imply that condition (ii) of Lemma 2.1 is also satisfied. Moreover, define

$$
H(x, \mu)=-\mu x+(1-\mu) \frac{1}{T} \int_{0}^{T}[e(t)-g(t, x)] d t ;
$$

in view of (3.4), we obtain that

$$
x H(x, \mu)<0 \text { for all } x \in \partial \Omega \cap \mathbb{R} \text { and } \mu \in[0,1],
$$

thus $H(x, \mu)$ is a homotopic transformation. From (3.4), together with the homotopic invariance theorem,

$$
\begin{aligned}
\operatorname{deg}(F, \Omega \cap \mathbb{R}, 0) & =\operatorname{deg}\left(\frac{1}{T} \int_{0}^{T}[e(t)-g(t, x)] d t, \Omega \cap \mathbb{R}, 0\right) \\
& =\operatorname{deg}(-x, \Omega \cap \mathbb{R}, 0) \neq 0 .
\end{aligned}
$$

This implies that condition (iii) of Lemma 2.1 is satisfied. Therefore, it follows from Lemma 2.1 and Remark 2.2 that there exists a $T$-periodic solution of (1.1). The uniqueness of $T$-periodic solutions of (1.1) is guaranteed by Lemma 2.4.

We now turn to asymptotic stability. Let $x_{0}(t)$ be the unique $T$-periodic solution of (1.1). Then we know from Lemma 2.5 and Remark 2.6 that

$$
\left|x_{0}\right|_{\infty} \leq M_{1} \text { and }\left|x_{0}^{\prime}\right|_{\infty} \leq M_{2} .
$$


Moreover, according to (2.23) and (2.24), it is easy to see that the linearized equation of (1.1) is

$$
y^{\prime \prime}(t)+f^{\prime}\left(x_{0}^{\prime}(t)\right) y^{\prime}(t)+g_{x}^{\prime}\left(t, x_{0}(t)\right) y(t)=0 .
$$

In order to show that $x_{0}(t)$ is asymptotically stable, Lemma 2.7 will be applied.

First, we show that (3.6) does not have real Floquet (or characteristic) multipliers. If not, then there is a real Floquet multiplier $\alpha$ and a nontrivial solution $y(t)$ of (3.6) such that $y(t+T)=\alpha y(t)$. Set $y(t)=\exp \left(-\frac{1}{2} \int_{0}^{t} f^{\prime}\left(x_{0}^{\prime}(s)\right) d s\right) u(t)$; then $u(t)$ is a non-trivial solution of the equation

$$
u^{\prime \prime}(t)+\left[g_{x}^{\prime}\left(t, x_{0}(t)\right)-\frac{1}{2} f^{\prime \prime}\left(x_{0}^{\prime}(t)\right) x_{0}^{\prime \prime}(t)-\frac{1}{4}\left(f^{\prime}\left(x_{0}^{\prime}(t)\right)\right)^{2}\right] u(t)=0
$$

with the Floquet multiplier $\beta=\alpha \exp \left(\frac{1}{2} \int_{0}^{T} f^{\prime}\left(x_{0}^{\prime}(s)\right) d s\right)$ (that is, $\left.u(t+T)=\beta u(t)\right)$.

Now we show that the following claim is true.

Claim. There exists a $t_{0} \in[0, T]$ such that

$$
u\left(t_{0}\right)=0 .
$$

Assume, by way of contradiction, that (3.8) does not hold. Then $u(t) \neq 0$ for all $t \in[0, T]$. Dividing (3.7) by $u(t)$ and integrating it from 0 to $T$, we obtain, noticing that $\int_{0}^{T} f^{\prime \prime}\left(x_{0}^{\prime}(t)\right) x_{0}^{\prime \prime}(t) d t=0$ and $u^{\prime}(0) / u(0)=u^{\prime}(T) / u(T)$, that

$$
\int_{0}^{T}\left(\frac{u^{\prime}(t)}{u(t)}\right)^{2} d t+\int_{0}^{T}\left[g_{x}^{\prime}\left(t, x_{0}(t)\right)-\frac{1}{4}\left(f^{\prime}\left(x_{0}^{\prime}(t)\right)\right)^{2}\right] d t=0,
$$

which together with (3.5) contradicts the condition $\left(\mathrm{H}_{6}\right)$ that $\int_{0}^{T}\left[g_{x}^{\prime}(t, x)-\right.$ $\left.\frac{1}{4}\left(f^{\prime}(y)\right)^{2}\right] d t>0$ for $x \in I_{1}$ and $y \in I_{2}$. This implies that (3.8) holds. Therefore we know that this $u(t)$ is a non-trivial solution of the Dirichlet boundary value problem

$$
\begin{gathered}
u^{\prime \prime}(t)+\left[g_{x}^{\prime}\left(t, x_{0}(t)\right)-\frac{1}{2} f^{\prime \prime}\left(x_{0}^{\prime}(t)\right) x_{0}^{\prime \prime}(t)-\frac{1}{4}\left(f^{\prime}\left(x_{0}^{\prime}(t)\right)\right)^{2}\right] u(t)=0, \\
u\left(t_{0}+T\right)=u\left(t_{0}\right)=0 .
\end{gathered}
$$

Since $\quad x_{0}^{\prime \prime}(t)=e(t)-f\left(x_{0}^{\prime}(t)\right)-g\left(t, x_{0}(t)\right)$, multiplying (3.10) by $u(t)$ and integrating it from $t_{0}$ to $t_{0}+T$, then, by (3.5) and $\left(\mathrm{H}_{7}\right)$,

$$
\begin{aligned}
& \int_{t_{0}}^{t_{0}+T} u^{\prime}(t)^{2} d t \\
& \quad=\int_{t_{0}}^{t_{0}+T}\left[g_{x}^{\prime}\left(t, x_{0}(t)\right)-\frac{1}{2} f^{\prime \prime}\left(x_{0}^{\prime}(t)\right) x_{0}^{\prime \prime}(t)-\frac{1}{4}\left(f^{\prime}\left(x_{0}^{\prime}(t)\right)\right)^{2}\right] u(t)^{2} d t \\
& \quad<\frac{1}{T^{2}} \int_{t_{0}}^{t_{0}+T} u(t)^{2} d t
\end{aligned}
$$

For convenience of use, we recall the Dirichlet-Poincaré inequality

$$
\int_{a}^{b}|f(t)|^{2} d t \leq(b-a)^{2} \int_{a}^{b}\left|f^{\prime}(t)\right|^{2} d t \quad \text { where } f \in C^{1} \text { and } f(a)=f(b)=0 .
$$


We immediately see that (3.11) contradicts the Dirichlet-Poincaré inequality. Therefore, (3.6) does not have real Floquet multipliers.

Next, we show that the characteristic exponents associated with (3.6) all have negative real parts. In order to do this, let us consider a system equivalent to (3.6):

$$
X^{\prime}(t)=A(t) X(t),
$$

where the vector function $X(t)=\left(x(t), x^{\prime}(t)\right)^{T}$ and $A(t)$ is the matrix function

$$
A(t)=\left[\begin{array}{cc}
0 & 1 \\
-g_{x}^{\prime}\left(t, x_{0}(t)\right) & -f^{\prime}\left(x_{0}^{\prime}(t)\right)
\end{array}\right] .
$$

Let $\alpha_{1}=e^{T \mu_{1}}$ and $\alpha_{2}=e^{T \mu_{2}}$ be the Floquet multipliers of (3.12) and $\mu_{1}$ and $\mu_{2}$ be the characteristic exponents associated with $\alpha_{1}$ and $\alpha_{2}$. Then it follows from the above discussion that $\alpha_{1}$ and $\alpha_{2}$ are a pair of complex conjugates. Applying Liouville's theorem (see [1, Problem 1, p. 285]),

$$
\alpha_{1} \alpha_{2}=e^{\int_{0}^{T} \operatorname{trace} A(t) d t}=e^{\int_{0}^{T}-f^{\prime}\left(x_{0}^{\prime}(t)\right) d t}
$$

and

$$
\operatorname{Re}\left(\mu_{1}\right)=\operatorname{Re}\left(\mu_{2}\right)=\frac{1}{2} \operatorname{Re}\left(\mu_{1}+\mu_{2}\right)=\frac{1}{2 T} \ln \left(\alpha_{1} \alpha_{2}\right)=\frac{1}{2 T} \int_{0}^{T}-f^{\prime}\left(x_{0}^{\prime}(t)\right) d t .
$$

By assumption $\left(\mathrm{H}_{6}\right)$, which says that $f^{\prime}(y)>0$ for $y \in I_{2}$, (3.14) leads to $\operatorname{Re}\left(\mu_{1}\right)=$ $\operatorname{Re}\left(\mu_{2}\right)<0$.

Finally, applying Lemma 2.7, we obtain that $x_{0}(t)$ is asymptotically stable. This completes the proof of Theorem 1.1.

REMARK 3.1. If $f(0) \neq 0$, the problem of the existence, uniqueness and asymptotic stability of $T$-periodic solutions to (1.1) can be converted into the equation

$$
x^{\prime \prime}(t)+f_{1}\left(x^{\prime}(t)\right)+g(t, x(t))=e_{1}(t),
$$

where $f_{1}\left(x^{\prime}(t)\right)=f\left(x^{\prime}(t)\right)-f(0), e_{1}(t)=e(t)-f(0)$. As $f_{1}(0)=0,(3.15)$ can be studied by means of Theorem 1.1 in this paper.

\section{An illustrative example}

In this section, we apply the main results obtained in previous sections to an example.

EXAMPLE 4.1. Consider the existence, uniqueness and asymptotic stability of $2 \pi-$ periodic solutions of the Rayleigh type equation

$$
x^{\prime \prime}(t)+\frac{1}{\pi^{4}} \arctan x^{\prime}(t)+\frac{1}{\pi^{4}}\left(\cos ^{2} t+1\right) x(t)=\frac{1}{\pi^{3}} \sin t,
$$

where $T=2 \pi$. Here $f\left(x^{\prime}\right)=\left(1 / \pi^{4}\right) \arctan x^{\prime}, g(t, x)=\left(1 / \pi^{4}\right)\left(\cos ^{2} t+1\right) x$ and $e(t)=\left(1 / \pi^{3}\right) \sin t$. 
ProOF. For convenience, let $\pi=3.1416$. Set $C_{1}=\left(1 / \pi^{4}\right), C_{2}=\left(2 / \pi^{4}\right)$ and $D_{1}=2 \pi$. Then it is easy to check that conditions $\left(\mathrm{H}_{1}\right)-\left(\mathrm{H}_{5}\right)$ of Theorem 1.1 are satisfied. Now we check that $\left(\mathrm{H}_{6}\right)$ and $\left(\mathrm{H}_{7}\right)$ also hold. According to Theorem 1.1, we can compute that $G=0, M_{0}=0.4376, M_{1}=6.8317, M_{2}=0.5485$, $I_{1}=[-6.8317,6.8317]$ and $I_{2}=[-0.5485,0.5485]$.

For $x \in I_{1}$ and $y \in I_{2}$,

$$
\begin{aligned}
\int_{0}^{2 \pi}\left[g_{x}^{\prime}(t, x)-\frac{1}{4}\left(f^{\prime}(y)\right)^{2}\right] d t & =\int_{0}^{2 \pi}\left[\frac{1}{\pi^{4}}\left(\cos ^{2} t+1\right)-\frac{1}{4 \pi^{8}\left(1+y^{2}\right)^{2}}\right] d t \\
& \geq \int_{0}^{2 \pi}\left[\frac{1}{\pi^{4}}\left(\cos ^{2} t+1\right)-\frac{1}{4 \pi^{8}}\right] d t \\
& =\frac{3}{\pi^{3}}-\frac{1}{2 \pi^{7}}>0
\end{aligned}
$$

and

$$
f^{\prime}(y)=\frac{1}{\pi^{4}\left(1+y^{2}\right)}>0,
$$

which imply that $\left(\mathrm{H}_{6}\right)$ holds.

On the other hand,

$$
\begin{aligned}
g_{x}^{\prime}(t, x)-\frac{1}{2} f^{\prime \prime}(y)[e(t)-f(y)-g(t, x)]-\frac{1}{4}\left(f^{\prime}(y)\right)^{2} \\
=\frac{1}{\pi^{4}}\left(\cos ^{2} t+1\right)+\frac{y}{\pi^{4}\left(1+y^{2}\right)^{2}}\left[\frac{1}{\pi^{3}} \sin t-\frac{1}{\pi^{4}} \arctan y-\frac{1}{\pi^{4}}\left(\cos ^{2} t+1\right) x\right] \\
\quad-\frac{1}{4 \pi^{8}\left(1+y^{2}\right)^{2}} \\
<\frac{2}{\pi^{4}}+\frac{1}{2 \pi^{4}} \times\left[\frac{1}{\pi^{3}}+\frac{1}{\pi^{4}} \times \frac{\pi}{2}+\frac{2}{\pi^{4}} \times 6.8317\right] \\
\approx 0.0216<\frac{1}{T^{2}}=\frac{1}{4 \pi^{2}} \approx 0.0253,
\end{aligned}
$$

which implies that $\left(\mathrm{H}_{7}\right)$ holds. Hence (4.1) has a unique $2 \pi$-periodic solution which is asymptotically stable.

REMARK 4.2. It is easy to see that the results in [1, 4, 7-11, 13-18] and the references therein cannot be applied to (4.1) in order to guarantee the existence, uniqueness and asymptotic stability of $2 \pi$-periodic solutions, so our results are new and complement the existing results.

\section{Acknowledgements}

The authors are grateful to the editor and referee for their careful reading of the manuscript and helpful suggestions on this work. 


\section{References}

[1] H. Chen and Y. Li, 'Rate of decay of stable periodic solutions of Duffing equations', J. Differential Equations 236 (2007), 493-503.

[2] E. A. Coddington and N. Levinson, Theory of Ordinary Differential Equations (McGraw-Hill, New York, 1955).

[3] N. H. Fletcher, 'Autonomous vibration of simple pressure-controlled valves in gas flows', J. Acoust. Soc. Am. 93 (1993), 2172-2180.

[4] R. Gaines and J. Mawhin, Coincidence Degree, and Nonlinear Differential Equations, Lecture Notes in Mathematics, 568 (Springer, Berlin, 1977).

[5] G. H. Hardy, J. E. Littlewood and G. Pólya, Inequalities (Cambridge University Press, London, 1964).

[6] W. L. Keith and R. H. Rand, 'Dynamics of a system exhibiting the global bifurcation of a limit cycle at infinity', Int. J. Nonlinear. Mech. 20 (1985), 325-338.

[7] A. C. Lazer and P. J. McKenna, 'On the existence of stable periodic solutions of differential equations of Duffing type', Proc. Amer. Math. Soc. 110 (1990), 125-133.

[8] Y. Li and L. Huang, 'New results of periodic solutions for forced Rayleigh-type equations', J. Comput. Appl. Math. 221 (2008), 98-105.

[9] S. Lu and W. Ge, 'Some new results on the existence of periodic solutions to a kind of Rayleigh equation with a deviating argument', Nonlinear Anal. 56 (2004), 501-514.

[10] S. Lu, W. Ge and Z. Zheng, 'Periodic solutions for a kind of Rayleigh equation with a deviating argument', Appl. Math. Lett. 17 (2004), 443-449.

[11] R. Manásevich and J. Mawhin, 'Periodic solutions for nonlinear systems with $p$-Laplacian-like operators', J. Differential Equations 145 (1998), 367-393.

[12] J. Mawhin, 'Periodic solutions of some vector retarded functional differential equations', J. Math. Anal. Appl. 45 (1974), 588-603.

[13] R. Ortega, 'Stability and index of periodic solutions of an equation of Duffing type', Boll. Unione Mat. Ital. Sez. B Artic. Ric. Mat. (7) 3 (1989), 533-546.

[14] T. Shimizu, 'Limit cycles and constants of motion in dissipative systems', Phys. Lett. A 69 (1979), 379-381.

[15] T. Shimizu and N. Morioka, 'Chaos and limit cycles in the Lorenz model', Phys. Lett. A 66 (1978), 182-184.

[16] J. W. Strutt [Lord Rayleigh], Theory of Sound, Volume 1 (Dover Publications, New York, 1877), reissued 1945.

[17] G. Q. Wang and S. S. Cheng, 'A priori bounds for periodic solutions of a delay Rayleigh equation', Appl. Math. Lett. 12 (1999), 41-44.

[18] Y. Zhou and X. Tang, 'On existence of periodic solutions of Rayleigh equation of retarded type', J. Comput. Appl. Math. 203 (2007), 1-5.

YONG WANG, School of Sciences, Southwest Petroleum University, Chengdu, Sichuan 610500, People's Republic of China

e-mail: odinswang@yahoo.com.cn, higher80@163.com

XIAN-ZHI DAI, School of Electronics and Information Engineering, Southwest Petroleum University, Chengdu, Sichuan 610500, People's Republic of China 